\title{
Effect of Light Intensity on Diurnal Sleep-Wake Distribution in Young and Old Rats
}

\author{
WIL WITTING, ${ }^{1}$ MAJID MIRMIRAN, NICO P.A. BOS AND DICK F. SWAAB \\ Netherlands Institute for Brain Research, Meibergdreef 33, 1105 AZ Amsterdam, The Netherlands
}

Received 28 May 1992; Accepted 17 June 1992

\begin{abstract}
WITTING, W., M. MIRMIRAN, N. P. A. BOS AND D. F. SWAAB. Effect of light intensity on diurnal sleep-wake distribution in young and old rats. BRAIN RES BULL 30(1/2) 157-162, 1993.-During the aging process, the amplitude of the circadian rhythms of many physiological variables is reduced. It has been hypothesized that increasing light intensity during the light phase of the light-dark cycle might result in a reduction of age-related changes in the circadian rhythms. Indeed, in the present sleepwake study in young and old rats it was found that (a) various parameters, such as the light-dark differences and total amounts of each behavioral state responded positively to changes in environmental light intensity (i.e., age-related trends were reversed), (b) in both age groups, the logarithm of light intensity appeared to have a linear dose-response relationship with light-dark differences of the sleep-wake states, (c) the light-dark difference of active wakefulness and quiet sleep of old rats under high light intensity were comparable to those of young rats under low light intensity. The results of the present study suggest that, under appropriate conditions, light could be of clinical use in reducing age-related circadian sleep disturbances in humans. This may, in turn, reduce the use of sedatives in elderly people.
\end{abstract}

Circadian rhythms Sleep-wakefulness Aging Alzheimer's disease Light therapy Rat

THE pervasive influence of light on circadian rhythms has been the interest of a large number of studies. Under constant environmental light, animals free run (i.e., exhibit day-night rhythms of approximately $24 \mathrm{~h}$ ) with both the period and amplitude of their rhythms being functions of the intensity of illumination (1). Furthermore, under a light-dark cycle the shapes of rhythms may be altered by modulation of the intensity of light $(2,17)$. These findings suggest that, in addition to being a "Zeitgeber," light is also an important modulator of circadian rhythms.

The profound influence of light on circadian systems has given rise to therapeutical interventions in two fields. First, patients suffering from seasonal affective disorders respond positively to light therapy, which has now become clinical practice in various countries (10). Second, in jet lag and shift work the period of adaptation can be minimized by application of light therapy $(5,6)$.

In geriatrics, disturbed circadian rhythms are an important reason for hospital admission (14) and may lead to an increased number of prescriptions for sedating drugs in the elderly (11) and increased workload for caregivers. During the aging process, several characteristics of circadian rhythms are subject to change. The most prominent and best-documented age-related change in circadian rhythms is the reduction in amplitude of various rhythms, including those of locomotor activity (21), feeding and drinking, temperature, various hormones, and sleep-wakefulness [see (19) for a review]. As changes in circadian amplitude can also be induced by changes in environmental lighting conditions, we have suggested (21) that light could be used to partially overcome age-related effects on circadian rhythms. The age-related effects on circadian rhythms that have been documented in humans are similar to those reported in the literature on rodents (19). Therefore, to determine whether light "therapy" could possibly be of use in the treatment of age-related circadian sleep disturbances the effect of different light intensities on the circadian sleep-wake rhythm was studied in both young and old rats.

METHOD

Animals

Eleven young adult (age: $6-8$ months old; weight \pm SEM: $379.5 \pm 6.3 \mathrm{~g})$ and 7 old (30-32 months old; $442.7 \pm 16.8 \mathrm{~g}$ ) male Brown-Norway rats (TNO Rijswijk) were anesthetized with Hypnorm ( $10 \mathrm{mg}$ fluanison $+2 \mathrm{mg}$ fentanyl $/ \mathrm{ml}$; $0.05-0.10 \mathrm{ml} /$ $\mathrm{kg}$ ) to allow implantation of electroencephalogram (EEG) and electromyogram (EMG) electrodes for chronic sleep-wake recordings. Two stainless steel screw electrodes were placed approximately $2 \mathrm{~mm}$ anterior to the bregma and lambda, respectively, and $2 \mathrm{~mm}$ left from the sagittal sinus for recording of EEG. Two double-twisted $100-\mu \mathrm{m}$ insulated nichrome wire electrodes were placed into the dorsal neck muscles for EMG recording. At least 1 week after surgery, rats were transferred to sound-proof sleep cages $(390 \times 272 \times 495 \mathrm{~mm}$; ambient tem-

${ }^{1}$ To whom requests for reprints should be addressed. 
perature $20-22^{\circ} \mathrm{C}$ ), where they were individually housed and given another week for adaptation.

\section{Experimental Conditions}

Both before and during the experiment, rats were kept under a 12L:12D cycle (lights on 7:00 a.m., lights off 7:00 p.m.). During the 15 days of the experiment, animals were exposed to five different light intensities during the light phase of the day. The maximum light intensities during the light phase measured in the middle of the cage at a height of $10 \mathrm{~cm}$, were $3.5,11.5,39$, 132, and 445 (i.e., approximately 3.39 to the power of 1, 2, 3, $4,5)$ lux. Each of these light intensities was maintained for 3 days. The light source (13498 Philips, 24 V/21 W) was affixed outside the cage to prevent masking effects due to temperature fluctuations caused by the heat of the lamp. A dim red light was maintained day and night at an intensity of approximately 0.1 lux.

\section{Data Acquisition}

A Tandon PC-AT computer and a CED-1401 AD converter were used for data acquisition. A new on-line sleep-wake classification program was used to determine the behavioral states with a sampling rate of $0.1 \mathrm{~Hz}$. It combines averaged EEG power spectra with integrated EMG activity in a teacher-supervised pattern classification algorithm to discriminate among four behavioral states: active wakefulness, quiet wakefulness, quiet sleep, and paradoxical sleep. If the integrated EMG level exceeded a preset level, active wakefulness was scored. Otherwise, one of the other sleep-wake stages was assigned depending upon the frequency distribution of the EEG. Full details of the procedure will be published elsewhere.

\section{Statistics}

Of the 3-day period at each light intensity, only days 2 and 3 were used for further analysis. On day 1, cages were cleaned and food and water refreshed, a procedure that took about 5$10 \mathrm{~min}$ per cage. The light-dark difference (as a measure of amplitude) and the total amounts of the different behavioral states per day and for the light and dark phases separately were calculated. Linear regression and Pearson's product moment correlation analysis were done on population means, and analysis of variance (ANOVA) with repeated measurements was performed on the individual light-dark differences and totals. To establish how changes in light intensity affected the distribution of each sleep-wake state within the 24-h day, days with the highest and lowest light intensities were also compared in 4-h blocks by means of paired $t$ tests.

\section{RESULTS}

Clear light-dark differences were observed in both age groups for active wakefulness, AW, $F(1,16)=412.50, p<0.001$; quiet sleep, QS, $F(1,16)=702.74, p<0.001$; paradoxical sleep, PS, $F(1,16)=124.45, p<0.001$, but not for quiet wakefulness $(\mathrm{QW})$, where only a trend was observed, $F(1,16)=$ $3.99, p<0.1$.

\section{Effects of Aging}

The total amounts of the different behavioral states were not altered in aged rats, age $\times$ total: $\mathrm{AW}, F(1,16)=0.87$, ns; QS, $F(1,16)=0.93$, ns; PS, $F(1,16)=0.01$, ns; QW, $F(1,16)=$ 0.14 , ns; Figs. 1A-1D. In contrast, the light-dark differences of AW and QS were significantly reduced during aging, age $\times$ light-
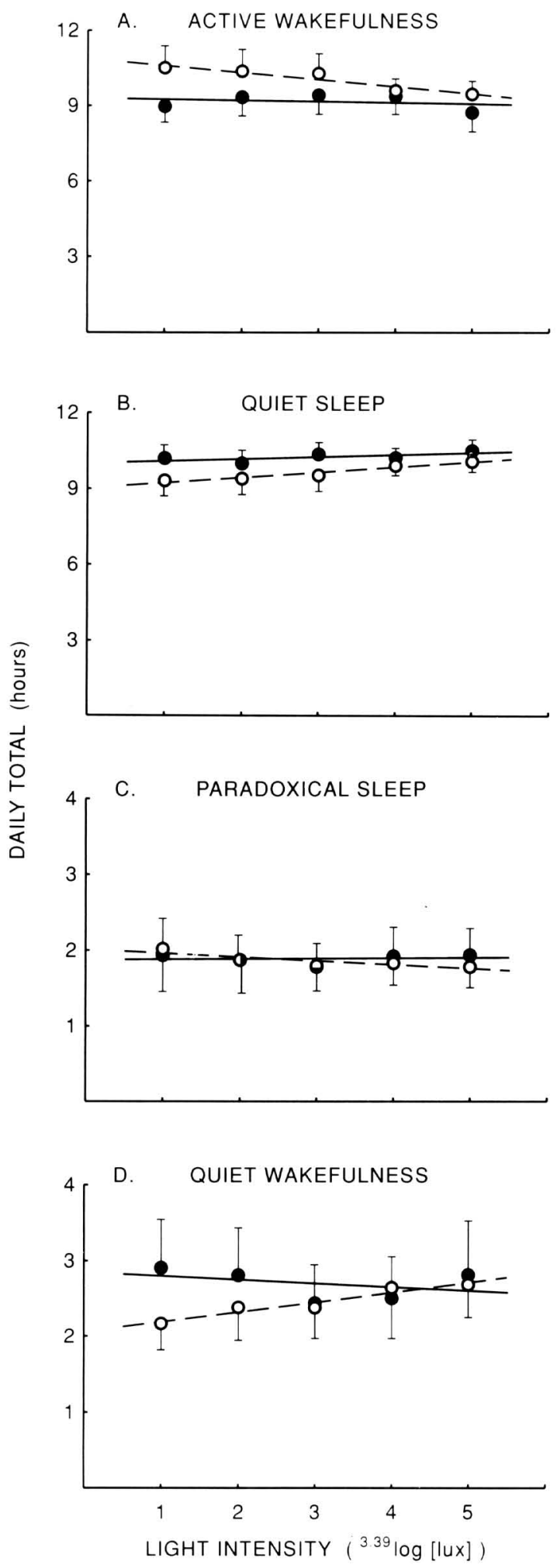

FIG. 1. Effect of light intensity on the daily totals of the four behavioral states (means \pm SEM; O: young rats; $\bullet$ : old rats). Although in both age groups the daily totals were affected by light, it seemed changes were more consistent in the younger group. As compared to the clear changes in light-dark differences, in old rats the minor changes in daily totals indicate a redistribution of the sleep-wake rhythm in this group. 
dark difference: AW, $F(1,16)=9.21, p<0.01$; QS, $F(1,16)=$ $10.47, p<0.01$. PS and QW remained unchanged, PS, $F(1,16)$ $=0.36$, ns; QW, $F(1,16)=1.31$, ns; Figs. $2 \mathrm{~A}-2 \mathrm{D}$.

\section{Effects of Light Intensity}

The light-dark differences of the population means of AW, QS, and PS were strongly correlated with light intensity: Figs. 2A-2D; Pearson's $r$ for AW, QS, PS, and QW in young rats: $0.93,0.99,0.86,0.60$; in old rats: $0.90,0.86,0.97,0.67$; critical $r$ for $\alpha_{0.05}(n=5)$, two tailed: \pm 0.88$)$. Results of the ANOVA were similar: There was a significant effect of light intensity on the light-dark difference of AW: intensity $\times$ light-dark difference, $F(4,13)=4.90, p<0.05$, QS, $F(4,13)=7.40, p<0.01$, and PS, $F(4,13)=4.32, p<0.05$, but not of QW, $F(4,13)=0.90$, ns. Total amount of AW was significantly decreased as a result of higher light intensity [intensity $\times$ total, $F(4,13)=3.71, p<$ 0.05 , whereas QS and QW tended to increase with light intensity: QS, $F(4,13)=2.71, p<0.1$; PS, $F(4,13)=1.56$, ns; QW, $F(4$, 13) $=2.56, p<0.1$; Figs. $1 \mathrm{~A}-1 \mathrm{D}$.

\section{Interaction Effects Between Age and Intensity}

No interaction effects on light-dark difference or totals were found by ANOVA; age $\times$ intensity $\times$ light-dark difference: $A W$, $F(4,13)=0.06$, ns; QS, $F(4,13)=0.140$, ns; PS, $F(4,13)=$ 0.27 , ns; QW, $F(4,13)=0.95$, ns; age $\times$ intensity $\times$ total: $\mathrm{AW}$, $F(4,13)=1.22$, ns; QS, $F(4,13)=0.56$, ns; PS, $F(4,13)=0.32$, ns; QW, $F(4,13)=2.16$, ns. The responses of young and old rats to the different light intensities were comparable, as was also apparent from the parallel regression lines (Figs. 2A-2D). On the other hand, correlation analysis on population means showed significant correlations in the young group only [Figs. 1A-1D; Pearson's $r$ for AW, QS, PS, and QW in young rats: $-0.94,0.97$, -0.74 , and 0.96 , respectively; in old rats: $-0.23,0.71,-0.06$, and -0.35 , respectively; critical $r$ for $\alpha_{0.05}(n=5)$, two tailed: \pm 0.88 ). Interestingly, the light-dark differences of AW and QS in old rats under the highest light intensity $(2.35 \pm 0.27$ and $1.92 \pm 0.17 \mathrm{~h}$, respectively) were comparable to those of young rats under the lowest light intensity $(2.45 \pm 0.13$ and $1.91 \pm$ $0.10 \mathrm{~h}$ ).

\section{Distribution Within the Light and Dark Phases}

According to ANOVA, the effect of light intensity on the circadian rhythms of the sleep-wake states appeared to be acute. The changes in light intensity primarily affected the total amounts of the sleep-wake stages during the light phase, with no significant after effects during the succeeding dark periods: intensity $\times$ light phase: AW, $F(4,13)=9.61, p<0.001$; QS, $F(4,13)=7.03, p$ $<0.01$; PS, $F(4,13)=2.45, p<0.01$; QW, $F(4,13)=1.84$, ns; intensity $\times$ dark phase: $\mathrm{AW}, F(4,13)=0.62$, ns; QS, $F(4,13)$ $=0.25$, ns; PS, $F(4,13)=1.46$, ns; QW, $F(4,13)=1.65$, ns. Although this may be true for a gross measure such as lightdark difference, closer examination made clear that both the light and dark phases are affected by changes in illumination (see below).

Comparison of the days with the highest and lowest light intensities in 4-h blocks revealed that the effect of light intensity was not solely restricted to the light phase. In fact, every behavioral state seemed to have its own specific response to changes in light intensity.

Active wakefulness (Fig. 3A-B). The effect of light on active wakefulness was strongest during the light phase, where the only significant differences were observed in both age groups. In young rats, the effects were most pronounced in the beginning and end
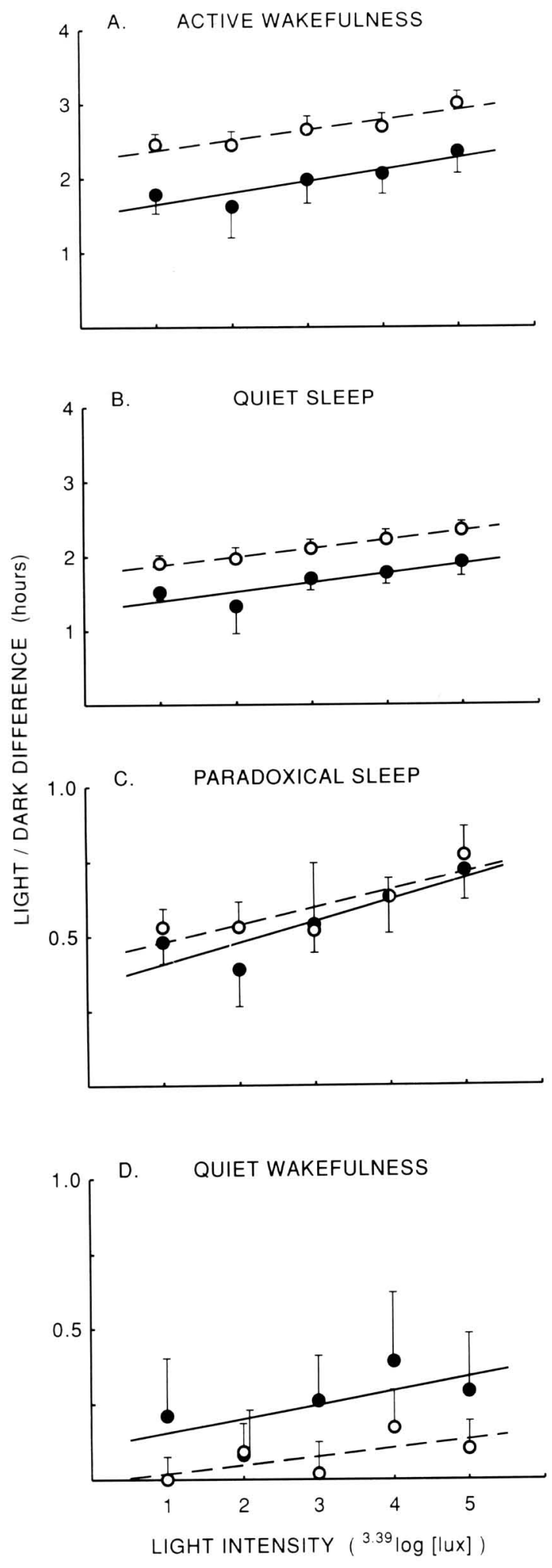

FIG. 2. Effect of light intensity on the light-dark differences of the four behavioral states (means \pm SEM; O: young rats; $\bullet$ : old rats). Especially for active wakefulness and quiet sleep, a clear dose-response relationship was present. The light-dark differences of active wakefulness and quiet sleep of old rats under high light intensity (intensity 5) are comparable to those of young rats under low light intensity (intensity 1). 


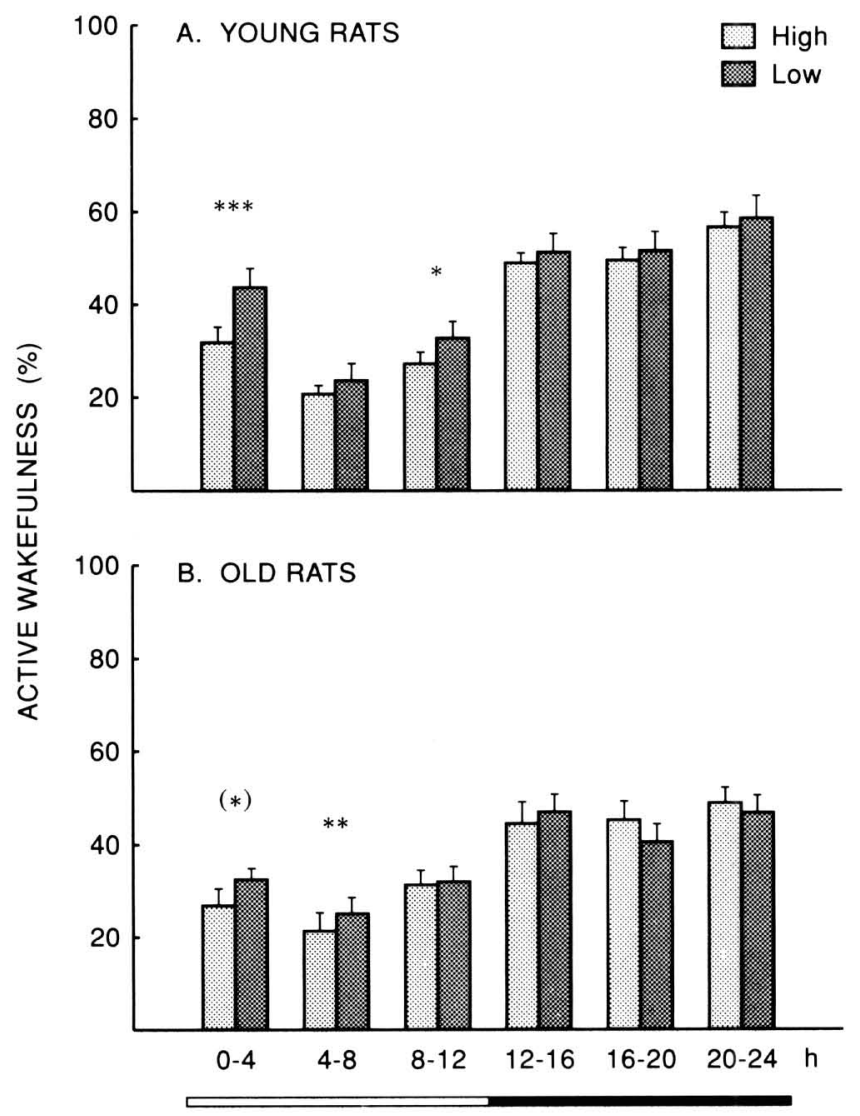

FIG. 3. Circadian profiles of active wakefulness of young (A) and old (B) rats during high (intensity 5) and low (intensity 1) light intensity. Data are presented per light intensity in six 4-h blocks. In both age groups, the effect of light was strongest during the light phase but the exact timing differed. For young rats, the changes were most significant during the first and third 4-h blocks, whereas in old rats the changes were found during the first and second blocks. Asterisks denote significance level of two tailed paired $t$ tests: ${ }^{* * *} p<0.001,{ }^{* *} p<0.01,{ }^{*} p<0.05,\left({ }^{*}\right) p<$ 0.10 .

of the light phase, whereas in old rats these effects were seen in the beginning and middle of the light phase.

Quiet sleep (Figs. 4A-B). In both age groups, significant changes were found during the light phase. In young rats under high light intensity, a significant increase was found during the first block and a tendency in the last block, whereas in old rats similar significant changes were found during the first and middle blocks. Old rats under low light intensity show an increased sleep tendency in the middle of their active phase (Fig. 4B). A significant increase and respective decrease was found from the fourth to fifth and fifth to sixth 4-h block. Under high light intensity, this fluctuation disappeared. A significant decrease was found during the middle of the dark phase under high as compared to low-light intensity (Fig. 4B).

Paradoxical sleep (Figs. 5A-B). No significant effects were found during the light phase in either age group. During the dark phase, significant differences were found in young rats. The middle and last blocks of the dark phase in young rats showed decreases in total amounts of paradoxical sleep under high light intensity.

Quiet wakefulness (Figs. $6 A-B$ ). The effect of changes in light intensity on quiet wakefulness seemed stronger in young than in old rats. Whereas in the young group significant reductions were observed in five of the six 4-h blocks, in the old group a reduction was only observed in the beginning of the light phase.

\section{DISCUSSION}

\section{Effects of Aging on the Distribution of Sleep-Wake States}

During aging, the circadian rhythms of many different variables are changed. The most frequently studied aspect of circadian rhythms, the amplitude or light-dark difference, is often reduced in aged animals (19). In the rat, the sleep-wake rhythm is similarly affected. Van Gool and Mirmiran $(18,20)$ showed a reduction of the amplitudes of wakefulness, quiet sleep, and paradoxical sleep. In our present study, we found similar changes for active wakefulness and quiet sleep but not for paradoxical sleep. However, from Fig. $2 \mathrm{C}$ it is clear that the presence or absence of significant differences with respect to this sleep stage may depend on ambient light intensity. Indeed, old rats under the lowest-light intensity showed a trend toward a smaller lightdark difference (one-tailed $t$-test: $p<0.10$ ) than young rats.

In humans, aging is often accompanied by a reduction in the "quality" of sleep. These changes include increased sleep latency, increased number of nocturnal awakenings, and increased

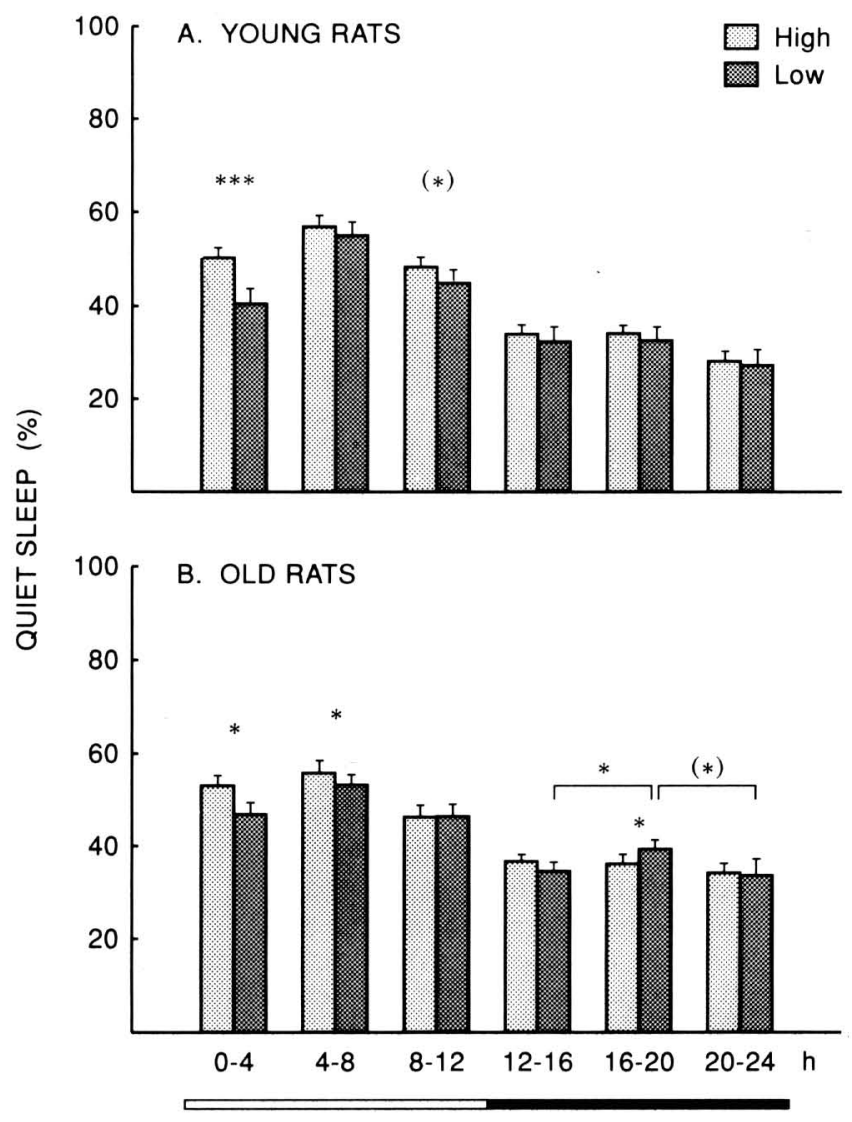

FIG. 4. Circadian profiles of quiet sleep of young (A) and old (B) rats during high and low light intensity. Old rats under low-light intensity show an increased sleep tendency in the middle of their active phase that disappeared under high light intensity. The changes found during the light phase were similar to those of active wakefulness. In young rats, they were most pronounced during the first and third blocks, as opposed to the first and second blocks in old rats. 


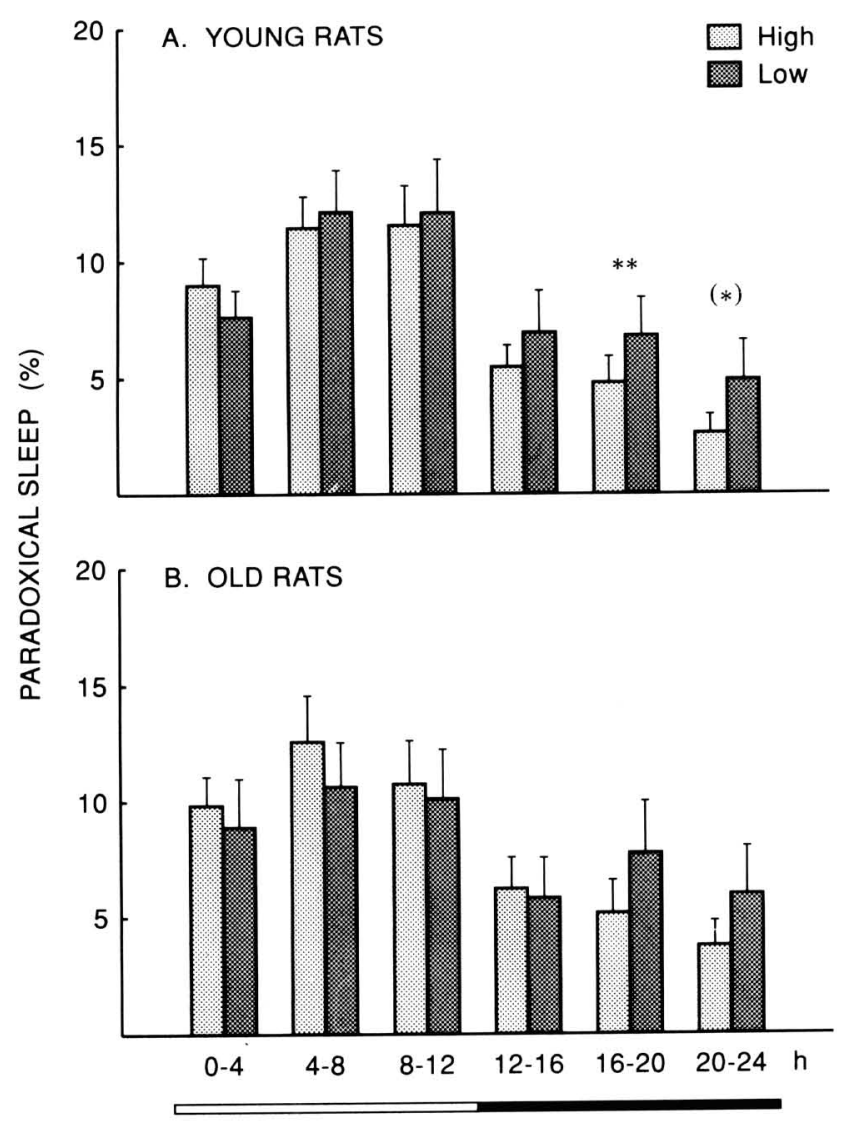

FIG. 5. Circadian profiles of paradoxical sleep of young (A) and old (B) rats during high and low light intensity. Surprisingly, the only significant changes were found in young rats during the dark phase in the fifth and sixth blocks.

number of daytime naps and result in an increased number of prescriptions for sedating drugs in elderly people (11). In turn, this has led to substantial efforts toward the development of new sedating drugs. In contrast, despite the vast amount of knowledge about the influence of light on circadian rhythms, and especially that of sleep-wake, this has led to only little clinical attention with respect to age-related sleep complaints.

Circadian rhythm disturbances in humans may be particularly pronounced $(12,21)$. As these disturbances also form an important reason for hospital admission (14), they may require closer study for social and economic, as well as medical, reasons.

Changes in various levels of the circadian system may contribute to the alterations in circadian rhythms (11). In the human, both the retina and the optic nerve, which provide the main source of light input to the suprachiasmatic nucleus (SCN), show degenerative changes in aging $(7,9,16)$. Furthermore, elderly people are exposed less to bright light than are younger people (3). Finally, with respect to the SCN, reductions in volume and cell number have been found in aging and, even more so, in patients suffering from Alzheimer's disease (15). In the latter group, the circadian rhythm disturbances tend to correlate with the severity of the disease (21). A decrease in the number of neurons containing vasopressin and vasoactive intestinal polypeptide in the SCN have also been reported in the aged rat $(4,13)$ whereas in the same animals cataracts are frequently observed (unpublished observation). Therefore, it is conceivable that both the amount of light transmitted through the retinohypothalamic tract and the processing of this information within the SCN are significantly impaired during aging. Consequently, the reported effects in the present article may be a result of increased light input to the circadian system, including the $\mathrm{SCN}$.

\section{Effects of Light on Distribution of sleep-wake States}

In 1939, Johnson (8) studied mice under constant illumination. In this study, decreases in amplitude and total amounts of movement activity were observed with increasing light intensity. Similarly, in our present study in rats under light-dark cycle the time spent asleep increased during the light phase and consequently the time spent awake decreased with increasing light intensity. However, a beneficial effect of light was observed with respect to increasing the circadian amplitude, which was comparable in young and old rats. Moreover, we found that old rats under low light intensity show an increased sleep tendency in the middle of their active phase that disappeared under high light intensity, whereas time asleep during the light phase of the day was augmented. This may imply that, at least in rats, the fluctuation in sleep tendency during the active phase is essentially the result of sleep loss during their inactive phase.

In general, theories and studies directed toward the practical implications of light as therapy lack a solid animal experimental formulation, making distinction between true and placebo effects
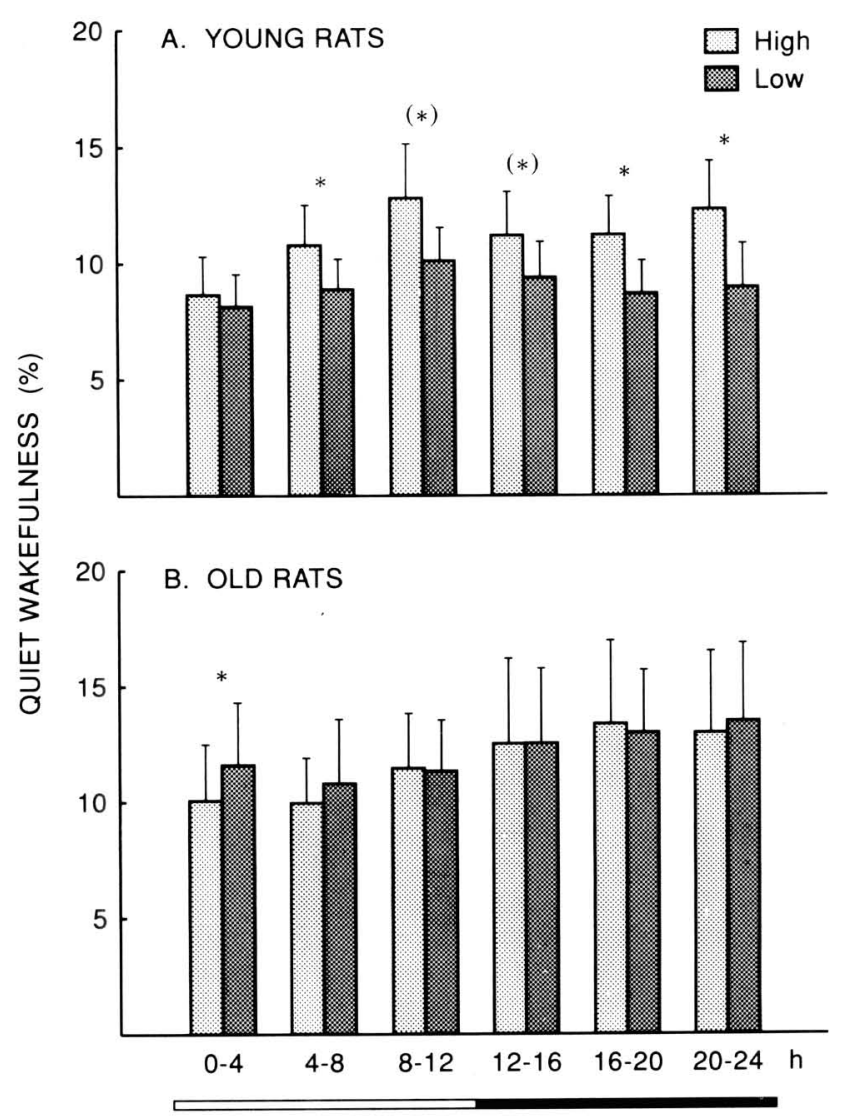

FIG. 6. Circadian profiles of quiet wakefulness of young (A) and old (B) rats during high and low light intensity. The effect of changes in light intensity seemed stronger in young than in old rats, as in young rats changes were seen in five of the six 4-h blocks, as opposed to only one significant change in old rats. 
difficult. For that reason, to explore the possibilities of light as a therapy in the treatment of age-related sleep disturbances in human the effect of changes in the level of daytime illumination on circadian sleep-wake rhythms was studied in young and old rats first. Although the results presented in this article appear promising, further study is required for understanding the nature of the effects of light. First, the reported effects in nocturnal rodents may not be directly applicable to diurnal animals, such as humans. Second, the disappearance or reduction of quantitative differences between young and old rats as a result of increased ambient light intensity may not necessarily imply the disappearance or reduction of qualitative differences. For example, it is important to know whether sleep is enhanced due to facilitation of sleep mechanisms or as a result of a reduction in sleep depth, two contrary processes that may both increase the total time spent asleep. This question may be solved by detailed EEG studies because sleep depth is considered to be reflected by total EEG power. Furthermore, our experimental design did not allow the examination of order effects, as the sequence of the different light intensities was not varied nor could we study any long-term effects.
The data cannot be interpreted in terms of an aversive effect of light in nocturnal animals. Although rats may prefer darkness to light when given a choice, there is no literature supporting the idea that nocturnal animals would avoid a less preferred situation by going to sleep.

In summary, the results of this study indicate once again the importance of illumination in sleep and waking. It was found that increasing daytime light intensity could reverse many of the age-related alterations in circadian sleep-wake rhythms in rats. Therefore, these findings suggest that, under appropriate conditions, light therapy might be of clinical use in reducing age-related sleep disturbances in humans, which may possibly lead to a reduction in the use of sedatives in elderly people.

\section{ACKNOWLEDGEMENTS}

We gratefully acknowledge the invaluable contributions of $\mathrm{M}$. A. Corner, R. Hoogland, R. Nooy, J. Overdijk, H. Stoffels, and D. van der Werf. Old animals were funded by the "NWO-bank voor oude proefdieren." This study was supported by STIPT, project "Ritmemeter," MTR 89026.

\section{REFERENCES}

1. Aschoff, J. Exogenous and endogenous components in circadian rhythms. Cold Spring Harbor Symp. Quantum Biol. 25:11-28; 1960.

2. Borbely, A. A. Sleep and motor activity of the rat during ultra-short light-dark cycles. Brain Res. 114:305-317; 1976.

3. Campbell, S. S.; Kripke, D. F.; Gillin, J. C.; Hubovcak, J. C. Exposure to light in healthy elderly subjects and Alzheimer patients. Physiol. Behav. 42:141-144; 1988.

4. Chee, C. A.; Roozendaal, B.; Swaab, D. F.; Goudsmit, E.; Mirmiran, M. Vasoactive intestinal polypeptide neuron changes in the senile rat suprachiasmatic nucleus. Neurobiol. Aging 9:307-312; 1988.

5. Czeisler, C. A.; Kronauer, R. E.; Allan, J. S.; Duffy, J. F.; Jewett, M. E.; Brown, E. N.; Ronda, J. M. Bright light induction of strong (type 0 ) resetting of the human circadian pacemaker. Science 244: 1328-1333; 1989.

6. Czeisler, C. A.; Johnson, M. P.; Duffy, J. F.; Brown, E. N.; Ronda J. M.; Kronauer, R. E. Exposure to bright light and darkness to treat physiologic maladaptation to night work. N. Engl. J. Med. 322:12531259; 1990.

7. Hinton, D. R.; Sadun, A. A.; Blanks, J. C.; Miller, C. A. Optic nerve degeneration in Alzheimer's disease. N. Engl. J. Med. 315:485-487; 1986.

8. Johnson, M. S. Effect of continuous light on periodic spontaneous activity of white-footed mice (peromyscus). J. Exp. Zool. 82:315$328 ; 1939$.

9. Katz, B.; Rimmer, S.; Iragui, V.; Katzman, R. Abnormal pattern electroretinogram in Alzheimer's disease: Evidence for retinal ganglion cell degeneration? Ann. Neurol. 26:221-225; 1987.

10. Lewy, A. J.; Sack, R. L.; Miller, L. S.; Hoban, T. M. Antidepressant and circadian phase-shifting effects of light. Science 235:352-354; 1987.

11. Miles, L. E; Dement, W. C. Sleep and aging. Sleep 3:119-220; 1980.
12. Okawa, M.; Mishima, K.; Shimizu, T.; Iijima, S.; Hishikawa, Y.; Hozumi, S.; Hori, H. Sleep-waking rhythm disorders and their phototherapy in elderly patients with dementia. Jpn. J. Psychiatry Neurol. 43:293-295; 1989.

13. Roozendaal, B.; Van Gool, W. A.; Swaab, D. F.; Hoogendijk, J. E.; Mirmiran, M. Changes in vasopressin cells of the rat suprachiasmatic nucleus with aging. Brain Res. 409:259-264; 1987.

14. Sanford, J. R. A. Tolerance of debility in elderly dependents by supporters at home: Its significance for hospital practice. Br. Med. J. 3:471-473; 1975.

15. Swaab, D. F.; Fliers, E.; Partiman, T. S. The suprachiasmatic nucleus in the human brain in relation to sex, age and senile dementia. Brain Res. 342:37-44; 1985.

16. Trick, G. L.; Barris, M. C.; Bickler-Bluth, M. Abnormal pattern electroretinograms in patients with senile dementia of the Alzheimer type. Ann. Neurol. 26:226-231; 1989.

17. Usui, S.; Takahashi, Y.; Honda, Y.; Ebihara, S. Circadian rhythms during various light-intensity cycles in rats. Physiol. Behav. 46:521$528 ; 1989$.

18. Van Gool, W. A.; Mirmiran, M. Age-related changes in the sleep pattern of male adult rats. Brain Res 279:394-398; 1983.

19. Van Gool, W. A.; Mirmiran, M. Aging and circadian rhythms. In: Swaab, D.F.; Fliers, E.; Mirmiran, M., eds. Progress in brain research. vol. 70. Aging of the brain and Alzheimer's disease. Amsterdam: Elsevier Science Publishers; 1986:255-279.

20. Van Gool, W. A.; Mirmiran, M. Effects of aging and housing in an enriched environment upon sleep-wake patterns in rats. Sleep 9: 335-347; 1986.

21. Witting, W.; Kwa, I. H.; Eikelenboom, P.; Mirmiran, M.; Swaab, D. F. Alterations in the circadian rest-activity rhythm in aging and Alzheimer's disease. Biol. Psychiatry 27:563-572; 1990. 